\title{
Distance between Speed Humps and Pedestrian Crossings: Does it Matter?
}

\author{
Charlotta J ohansson, Peter Rosander, Lars Leden \\ Luleå University of Technology, Sweden. \\ Charlotta.M.Johansson@Itu.se \\ T: +46920491867
}

\begin{abstract}
Speed humps are a common physical measure installed at pedestrian crossings to reduce vehicle speeds and therefore improve the safety of pedestrians at the crossing.

The aim of this study was to determine whether variations in distance between speed humps and pedestrian crossings contribute differently to the safety of pedestrians and cyclists, especially children and the elderly, and if so, how. Three sites in Sweden were studied, where vehicle speed and video filming at the site resulted in manually coded, road user behaviour of 1972 pedestrians and cyclists.
\end{abstract}

The humps were installed at distances of 5-10 m from the pedestrian crossing, ie about one or two car lengths. As vehicle speeds were somewhat lower at the pedestrian crossing when the distance between the speed hump to the pedestrian crossing was greater, and there were some positive aspects regarding the mobility of the pedestrians and cyclists, a greater distance of about $10 \mathrm{~m}$ or two car lengths is suggested. The present study only covers speed humps, but the same distance is also regarded as important when installing other types of physical measures to reduce vehicle speed.

\section{NTRODUCTI ON}

Actual vehicle speed should be a maximum of $30 \mathrm{~km} / \mathrm{h}$ where there is a risk of collision between vehicles and unprotected road users. At speeds of $50 \mathrm{~km} / \mathrm{h}$, the probability of pedestrian death is 5-8 times greater than at $30 \mathrm{~km} / \mathrm{h}$ (Ashton, 1982, Teichgräber, 1983, Waltz, 1983). Speed cushions are a common type of physical measure used to increase safety and the accessibility for pedestrians and cyclists at crossings and cycle routes in builtup areas. These measures are recorded as being very effective (Elvik and Vaa, 2004).

The aim of this study is to determine how various distances between speed cushions and crossings affect safety and accessibility for pedestrians and cyclists, especially children but also the elderly. The study only covers speed cushions, but it is likely that the same distance is suitable for the location of other types of speed reducing devices.

\section{METHOD}

The following methods of data capture were used: radar and laser measurement of vehicle speed, video recording of road user behaviour.

\section{Radar and laser measurement of vehicle speed}

Radar and laser measurements of free vehicle speed were taken at the pedestrian crossings, ie theoretical collision site, as well as speeds $12 \mathrm{~m}$ before the pedestrian crossing, the last point at which drivers could be expected to have started to decelerate. 


\section{Video recording of road user behaviour}

At each site, video recordings were made from different angles in order to capture the behaviour and encounters of various road users. Recordings of the crossings were made over two days with two cameras giving a general view of the crossing and close-ups of pedestrians and cyclists. Data entry from video recordings was carried out manually in Excel in accordance with the parameters given below. The parameters can be placed in three groups, (1) description of the pedestrian or the cyclist and their behaviour, (2) description of the vehicle and motorist behaviour, and (3) description of the interaction between them. By comparing $1.5 \mathrm{~h}$ of video material comprising 47 crossings, two observers had $83 \%$ agreement in behaviour coding. When the most difficult parameter to encode, pedestrian and cyclist head movement, was eliminated, agreement was 89\% (Johansson, 2001).

Codings were stratified so that behaviour for children (0-12 years), young people (13-19), elderly (over 64) and functionally disabled irrespective of age, was encoded. The behaviour of adults in the 20-64 age group was encoded if they were in the company of the above groups. Sites were videoed from 0730 - 0900 and for three successive hours in the interval 1300-1630. The parameters studied are given below.

(a) sex

(b) age

(c) mode of transport

(d) number of persons in group

(e) sex of oldest in group

(f) age of oldest in group

(g) whether they stop at edge of the pavement, kerb

(h) whether they stop on the refuge

(i) whether they cross at the crossing

(j) whether they cross diagonally or straight (k) pace before crossing

(I) pace on crossing first lane

(m) pace on crossing second lane

(n) pace after crossing

(o) traffic situation, where first car comes from

(p) whether vehicles from left give way

(q) whether vehicles from right give way

(r) long or short distance to speed cushion

(s) driver behaviour in giving way

(t) comments

Differences in results depending on long or short distances between speed cushion and pedestrian crossing, and speed cushion and cycle crossing, were analysed using the chisquared test, and are commented on at $95 \%$ significance.

\section{DESCRI PTI ON OF SI TES AND DATA CAPTURE}

Studies were made at three sites in Sweden, the Regementsgatan/Dragonstigen and Tessins Väg/Henrik Wranérs Gata crossings in Malmö, and a pedestrian and cycle crossing on Hultagatan in Borås. The sites were studied over different periods, see Table 1. A total of 1972 pedestrian and cyclist crossings were studied from a total of $62 \mathrm{~h}$ of film.

Table 1. Study sites and overall data.

\begin{tabular}{lccc}
\hline & Data & $\begin{array}{c}\text { Total number } \\
\text { hours }\end{array}$ & $\begin{array}{c}\text { Total number } \\
\text { observations }\end{array}$ \\
\hline Regementsgatan/Dragonstigen, Malmö & $2001-2006$ & 36 & 818 \\
Tessins Väg/Henrik Wranérs Gata, Malmö & 2006 & 9.5 & 437 \\
Hultagatan, Borås & 2002 & 16.5 & 717 \\
\hline Total & & $\mathbf{6 2}$ & $\mathbf{1 9 7 2}$ \\
\hline
\end{tabular}




\section{Regementsgatan/ Dragonstigen, Malmö}

There are two schools in the vicinity of the two study sites in Malmö, which means that many children pass Regementsgatan and Tessins Väg on their way to or from the school or during their free time. There are also many elderly and disabled people in the area, and there is bus traffic as well as some other heavy traffic such as goods vehicles. The crossing at Dragonstigen is a pedestrian and cycle crossing on a stretch with pedestrian and cycle crossings. There are speed cushions at approximately $5 \mathrm{~m}$ and $8.8 \mathrm{~m}(5.2 \mathrm{~m}$ in front of the cycle crossing) before the pedestrian crossing, see Fig 1 . The height of the speed cushions is about $100 \mathrm{~mm}$. White cobbles are used to indicate the pedestrian crossings, see Figs 2 and 3. The speed limit is $50 \mathrm{~km} / \mathrm{h}$, but the recommended speed is $30 \mathrm{~km} / \mathrm{h}$. Vehicle flow at the site was taken as the average annual daily traffic based on 2005 figures, or 9000 vehicles a day.

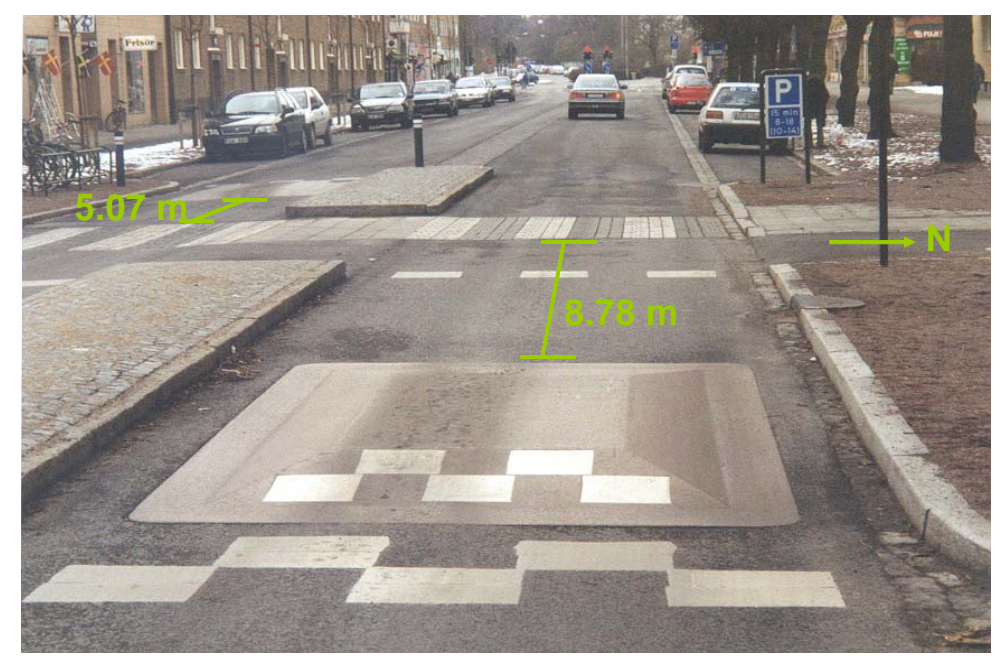

Fig 1. Dimensions of the Dragonstigen - Regementsgatan crossing looking west.

\section{Tessins Väg/ Henrik Wranérs gata, Malmö}

Tessins Väg goes through Ribersborg in the western part of central Malmö. The distance between the pedestrian crossing and the speed cushion is $4.2 \mathrm{~m}$ for traffic travelling north, and $10.2 \mathrm{~m}$ for vehicles travelling south-west. The distance between the pedestrian crossings on each side of the crossing is approximately $19 \mathrm{~m}$. Crossings and speed cushions are indicated with painted markings, and the height of the speed cushions is about $100 \mathrm{~mm}$. Signed speed limit through the crossing is $30 \mathrm{~km} / \mathrm{h}$ between 0700 and 1700 on weekdays, but not during the three summer months (16/6-14/8) when a $50 \mathrm{~km} / \mathrm{h}$ limit applies instead. Tessins Väg had a vehicle flow of 4000 vehicles per day in 2005.

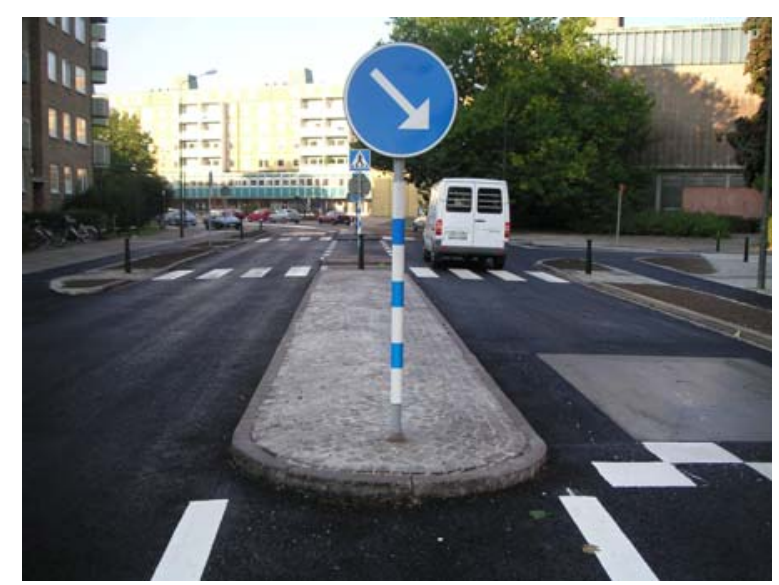

Fig 2. Details of the Tessins Väg/Henrik Wranérs gata crossing after reconstruction, looking southwest. 


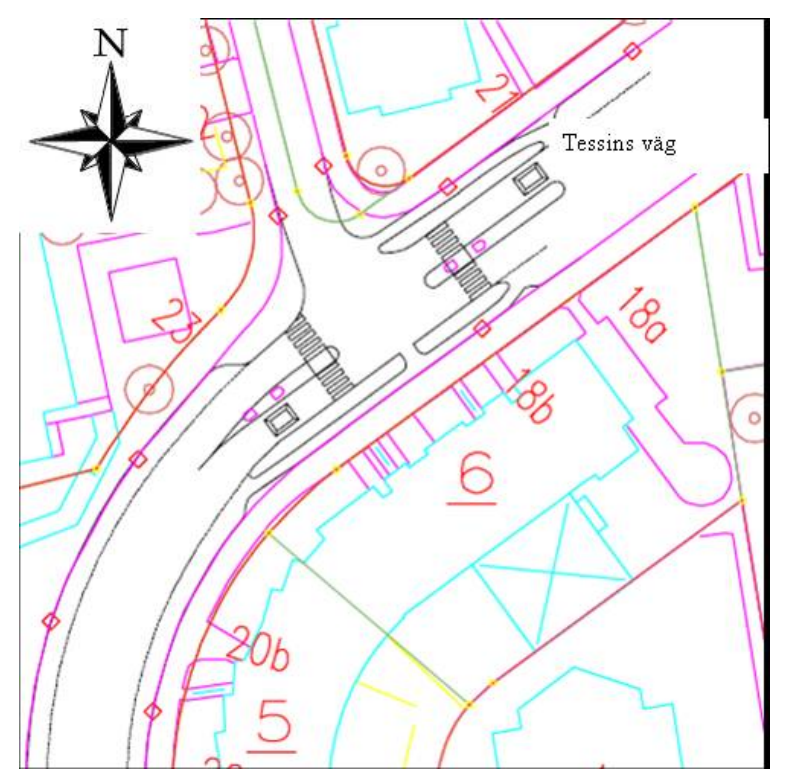

Fig 3. Plan of the Tessins Väg/Henrik Wranérs Gata crossing after reconstruction.

\section{Hultagatan, Borås}

The Hulta centre with junior and middle schools and businesses lies south of the area studied. Hultagatan had a daily vehicle flow of 5000 vehicles in 2001 . Signed speed limit is $50 \mathrm{~km} / \mathrm{h}$ but the recommended speed is $30 \mathrm{~km} / \mathrm{h}$. See Figs 4 and 5 for appearance and layout. The height of the speed cushions is $70 \mathrm{~mm}$.

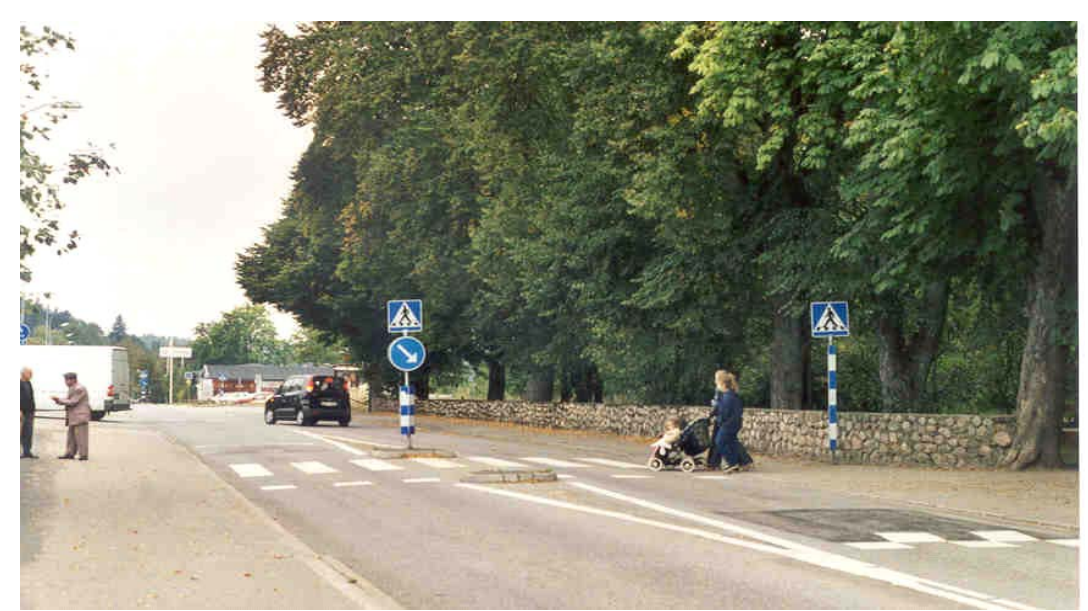

Fig 4. Crossing site with cycle passage on Hultagatan looking west.

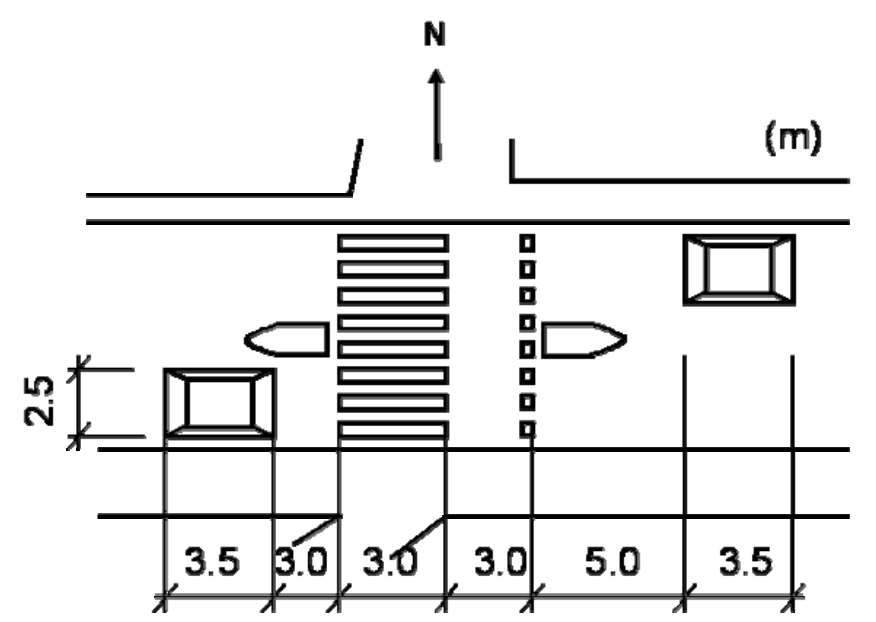

Fig 5. Diagram of crossing with cycle passage on Hultagatan. 
At two of the sites there were cycle crossings which meant that the distance to the pedestrian crossing was differently depending on the direction of the oncoming vehicular traffic. At the third site, the speed cushions are located at different distances from the pedestrian crossing. All three sites are equipped with central islands. The aim of the measures was to reduce vehicle speed at the conflict points with unprotected road users.

\section{EFFECT OF DI STANCES}

The analyses concern the effect on pedestrian, cyclist, and driver behaviour of the various distance between the speed cushion and crossing place in the different directions of the approaching vehicles. The pedestrian crossings that were analysed were those nearest to the speed cushion, and the distance between speed cushion and pedestrian crossing is expressed as long or short (PCR=pedestrian crossing). In cases where significant differences were shown for the respective road user group analysed, further analyses with tests to see if the differences also applied per site were carried out to ensure that the difference can be considered to apply generally.

\section{Total pedestrians}

In all, 1350 pedestrians were observed, and 802 (approximately 60\%) of these encountered a vehicle; $89 \%$ of all pedestrians crossed the road at the pedestrian crossing. The only significant difference shown was that pedestrians as a group were more often given way by the first car from the left if there was a greater distance between the crossing and the speed cushion, $50 \%$ at greater distances compared with $40 \%$ at shorter $(p<0.05)$, see Table 2 . Based on all 255 pedestrian observations from Dragonstigen, the tendency was the same, $50 \%$ compared with $43 \%$ (difference not significant), on Hultagatan (187 observations) there was a significant difference of $43 \%$ and $23 \%(p<0.01)$. On Tessins Väg, based on a total of 74 observations, the proportion given way by the first car from left was $64 \%$ when the distance was long, and $70 \%$ when the distance was short (difference not significant), i.e. just the opposite for the whole group of pedestrians in total, and for the other places. This may possibly be due to lower visibility at the short distance than at the longer distance.

Table 2. Pedestrian and driver behaviour depending on distances between left speed cushion and pedestrian crossing before the island for the pedestrian. $\mathrm{PCR}=$ pedestrian crossing.

\begin{tabular}{|c|c|c|}
\hline Given way by some motorists & $\mathrm{n}$ & $\%$ \\
\hline shorter distance left hump to PCR & 400 & 71 \\
\hline longer distance left hump to PCR & 325 & 74 \\
\hline \multicolumn{3}{|l|}{ Given way by any cars from the left } \\
\hline shorter distance left hump to PCR & 292 & 58 \\
\hline longer distance left hump to PCR & 224 & 60 \\
\hline \multicolumn{3}{|l|}{ Given way by first car from the left* } \\
\hline shorter distance left hump to PCR & 292 & 40 \\
\hline longer distance left hump to PCR & 224 & 50 \\
\hline \multicolumn{3}{|c|}{ Pedestrian waits at edge of the pavement when first car comes from the left } \\
\hline shorter distance left hump to PCR & 181 & 29 \\
\hline longer distance left hump to PCR & 193 & 28 \\
\hline \multicolumn{3}{|c|}{ Pedestrians who encounter first car from the left look in both directions at the kerb } \\
\hline shorter distance left hump to PCR & 98 & 49 \\
\hline longer distance left hump to PCR & 143 & 50 \\
\hline \multicolumn{3}{|c|}{ Pedestrians who encounter any cars look in some direction at the kerb } \\
\hline shorter distance left hump to PCR & 98 & 93 \\
\hline longer distance left hump to PCR & 143 & 93 \\
\hline
\end{tabular}

*Significant difference with 95\% probability. 
There was also a significant difference between the three sites in terms of the proportion given way by the first car from the left at short distances between speed cushion and the pedestrian crossing $(p<1.0 \mathrm{e}-6)$. This means that there was a significant difference in how often people were given way by the first car from the left when there was a short distance, but the trend was still similar, that there were fewer per site given way at short distances than at longer distances between the speed cushion and the pedestrian crossing. There was no significant difference between the sites when the distance between speed cushion and pedestrian crossing was long.

In other respects, the proportion of road user behaviour depending on different distances between speed cushion and pedestrian crossing was very similar. Just over $70 \%$ of all pedestrians were given way by some motorists, and approximately $60 \%$ were given way by some motorists from the left.

No significant differences were shown in the study of road user behaviour in the other lane, i.e. after the island (in other words, vehicles that came from the right seen from the pedestrian's perspective), see Table 3. Driver behaviour towards the pedestrian appears to be similar irrespective of whether the pedestrian is located at the edge of the pavement or on the island, with about $60 \%$ of all pedestrians given way irrespective of whether the car came from the right or left.

Table 3. Pedestrian and driver behaviour depending on distance between right speed cushion and crossing after the island for the pedestrian. PCR $=$ pedestrian crossing.

\begin{tabular}{|c|c|c|}
\hline Given way by some motorists & $\mathrm{n}$ & $\%$ \\
\hline SP shorter distance right hump to PCR & 316 & 74 \\
\hline longer distance right hump to PCR & 372 & 70 \\
\hline \multicolumn{3}{|l|}{ Given way by any cars from the right } \\
\hline SP shorter distance right hump to PCR & 259 & 64 \\
\hline longer distance right hump to PCR & 252 & 63 \\
\hline \multicolumn{3}{|l|}{ Given way by first car from the right } \\
\hline SP shorter distance right hump to PCR & 259 & 55 \\
\hline longer distance right hump to PCR & 252 & 56 \\
\hline \multicolumn{3}{|c|}{ Pedestrian stays at the island when first car comes from the right } \\
\hline shorter distance right hump to PCR & 102 & 3 \\
\hline longer distance right hump to PCR & 138 & 4 \\
\hline \multicolumn{3}{|c|}{ Pedestrians who encounter first car from the right look in both directions at the refuge } \\
\hline shorter distance right hump to PCR & 66 & 5 \\
\hline longer distance right hump to PCR & 105 & 4 \\
\hline \multicolumn{3}{|c|}{ Pedestrians who encounter first car from the right look in some direction at the refuge } \\
\hline shorter distance right hump to PCR & 66 & 56 \\
\hline longer distances right hump to PCR & 105 & 42 \\
\hline
\end{tabular}

21 of the 525 (4\%) pedestrians who did not encounter a car waited on the edge of the pavement, at the kerb, and none waited on the refuge island. Of those who did not encounter any vehicle, approximately 33\% looked in both directions at the edge of the pavement irrespective of journey direction, and more than $90 \%$ looked in some direction at the edge of the pavement. A few, approximately $3 \%$, looked in both directions on the island and approximately $30 \%$ looked in some direction on the island. 


\section{Children and elderly pedestrians}

In all, 371 children were observed, $224(60 \%)$ of whom encountered a car, and 147 did not. Of all child pedestrians, $92 \%$ crossed the road at the crossing. No significant differences were shown in the children's behaviour or vehicle driver behaviour in the different directions.

Twelve ( $8 \%$ ) of the 144 child pedestrians who did not encounter a car waited at the edge of the pavement without encountering a car. Of those who did not, approximately $48 \%$ looked in both directions at the edge of the pavement irrespective of journey direction, and approximately $76 \%$ looked in some direction at the edge of the pavement.

A total of 258 elderly pedestrians were observed, 161 (62\%) of whom encountered a car. No significant differences were observed in the behaviour of either the elderly pedestrians or the vehicle drivers from either direction. Of the 97 elderly pedestrians who did not encounter a car, two (approximately $2 \%$ ) waited at the edge of the pavement before they crossed the road. Of all elderly pedestrians, $96 \%$ crossed the road at the pedestrian crossing.

\section{Total cyclists}

For cyclists the analyses were carried out in the same way as for pedestrians, but for cyclists the road user behaviour is compared with long and short distances between speed cushions and the cycle crossing. However on the Tessins Väg site there is no cycle crossing, so distances are measured between the speed cushion and the pedestrian crossing, On Hultagatan the distance is approximately equal between the cycle crossings and the two speed cushions in each direction. If the measurements from Hultagatan are ignored the results are similar.

A total of 613 cyclists was observed, and $356(58 \%)$ of them encountered a car. A total of $84 \%$ of all cyclists crossed the road at the pedestrian crossing or cycle crossing, but the results below refer to all cyclists. Two of the 252 cyclists who did not encounter a car waited at the edge of the pavement. Of those who did not encounter a car, approximately $50 \%$ looked in both directions at the edge of the pavement irrespective of journey direction, and $80 \%$ looked in some direction at the edge of the pavement.

Cyclists were given way by motorists significantly more often when the distance between speed cushion and cycle crossings was longer, $75 \%$ compared to $57 \%$, see Table 4 . They were also given way by the first car from the left significantly more often when the distance was longer. Based on a total of 231 cyclist observations from Dragonstigen, the tendency was the same, $82 \%$ compared to $58 \%$ (significant difference), but on Hultagatan (96 observations) the figures were $62 \%$ and $57 \%$ (not significant). On Tessins Väg, only 13 observations were made in total. Cyclists were also significantly more often given way by the first motorist when the distance between speed cushion and cycle crossing was longer ( $p<$ 0.05), see Table 4 . The trend was also the same for cyclists split over the various places.

The cyclists were significantly more often given way by the first car from the left when they were at the edge of the pavement and the distance to the speed cushion was longer, but were also significantly more often given way by some motorists when the distance to the speed cushion was shorter when they were waiting at the island $(p<0.01)$. A plausible explanation is that the cyclists in question were given way by the first motorist they encountered, which is assumed to be from the left, and then it is reasonable that also motorists from the right give way, since those from the left have already waited or slowed up. That behaviour was not detected in the case of pedestrians. 
Table 4. Cyclist and driver behaviour depending on distance between left speed cushion and pedestrian crossing before the island for cyclists (CCR = cycle crossing).

\begin{tabular}{lcr}
\hline Given way by some motorists* & $n$ & $\%$ \\
\hline shorter distance left hump to CCR & 167 & 57 \\
longer distance left hump to CCR & 173 & 75 \\
\hline Given way by any car from the left & 128 & 47 \\
\hline shorter distance left hump to CCR & 127 & 57 \\
longer distance left hump to CCR & 128 & 37 \\
\hline Given way by the first car from the left* & 127 & 51 \\
\hline shorter distance left hump to CCR & 116 & 34 \\
longer distance left hump to CCR & 90 & 42 \\
\hline Cyclist stays at edge of the pavement when first car comes from the left & 54 \\
\hline shorter distance left hump to CCR & 102 & 63 \\
\hline longer distance left hump to CCR & 72 & 93 \\
\hline Cyclist who encounters first car from the left looks in both directions at the kerb & 94 \\
\hline shorter distance left hump to CCR & 102 & \\
\hline longer distance left hump to CCR & 72 & \\
\hline Cyclist who encounters first car from the left looks in some direction at the kerb & \\
\hline shorter distance left hump to CCR & & \\
longer distance left hump to CCR &
\end{tabular}

Table 5. Cyclist and driver behaviour depending on distance between right speed cushion and pedestrian crossing after the island for cyclists ( $C C R=$ cycle crossing).

\begin{tabular}{|c|c|c|}
\hline Given way by some motorists* & $\mathrm{n}$ & $\%$ \\
\hline shorter distance right hump to CCR & 174 & 74 \\
\hline longer distance right hump to $C C R$ & 169 & 57 \\
\hline \multicolumn{3}{|l|}{ Given way by any cars from the right } \\
\hline shorter distance right hump to CCR & 123 & 63 \\
\hline longer distance right hump to $\mathrm{CCR}$ & 110 & 53 \\
\hline \multicolumn{3}{|c|}{ Given way by the first car from the right } \\
\hline shorter distance right hump to CCR & 123 & 59 \\
\hline longer distance right hump to $\mathrm{CCR}$ & 110 & 47 \\
\hline \multicolumn{3}{|c|}{ Cyclist stays at island when first car comes from the right } \\
\hline shorter distance right hump to CCR & 82 & 2 \\
\hline longer distance right hump to CCR & 104 & 1 \\
\hline \multicolumn{3}{|c|}{ Cyclist who encounters first car from the right looks in both directions at the refuge } \\
\hline shorter distance right hump to CCR & 63 & 5 \\
\hline longer distance right hump to CCR & 99 & 1 \\
\hline \multicolumn{3}{|c|}{ Cyclist who encounters first car from the right looks in some direction at the refuge } \\
\hline shorter distance right hump to CCR & 63 & 27 \\
\hline longer distance right hump to CCR & 99 & 30 \\
\hline
\end{tabular}

*Significant difference with $95 \%$ probability.

\section{Cycling children and elderly}

In all, 134 cycling children were observed, and 86 (64\%) of them encountered a vehicle. Therefore, $92 \%$ of all cycling children crossed the road at the pedestrian or cycle crossing.

As for all cyclists, children were more often given way when the distance between the speed cushion and pedestrian crossing was longer, $80 \%$ as opposed to $51 \%$ at shorter distances ( $p$ $<0.001$ ), see Table 6 . Again, like the 'all cyclists' group, children were obviously more often given way by motorists coming from the right when the distance was shorter, $78 \%$ as 
opposed to $58 \%$ at longer distances. The total of elderly cyclists observed was 33 , which is too small for further analysis.

Tabell 6. Child cyclist and driver behaviour depending on distance between left speed cushion and pedestrian crossing before the island for cyclists (CCR = cycle crossing).

\begin{tabular}{lcc} 
Given way by some motorists* & $\mathrm{n}$ & $\%$ \\
\hline shorter distance left hump to CCR & 35 & 51 \\
longer distance left hump to CCR & 51 & 80 \\
\hline Given way by any car from the left & & 67 \\
\hline shorter distance left hump to CCR & 39 & 60 \\
longer distance left hump to CCR & 67 & 62 \\
\hline Given way by the first car from the left & 39 & 52 \\
\hline shorter distance left hump to CCR & 67 & 30 \\
longer distance left hump to CCR & 27 \\
\hline Cyclist stays at the kerb when first car comes from the left & 27 & 33 \\
\hline shorter distance left hump to CCR & 30 & 50 \\
longer distance left hump to CCR & 18 & 89 \\
\hline Cyclist who encounters first car from the left looks in both directions at the kerb \\
\hline shorter distance left hump to CCR & 20 & 95 \\
longer distance left hump to CCR & 18 & 20 \\
\hline Cyclist who encounters first car from the left looks in some direction at the kerb \\
\hline shorter distance left hump to CCR \\
longer distance left hump to CCR
\end{tabular}

*Significant difference with $95 \%$ probability.

\section{Vehicle speeds}

The two sites in Malmö with different distances between speed cushion and pedestrian crossing had similar measured values of motor vehicle speed at the pedestrian crossing in both directions. What was unexpected was that, when the distance to the pedestrian crossing was longer, speed was somewhat lower (not statistical significant) at the pedestrian crossing, just over $22 \mathrm{~km} / \mathrm{h}$ at longer distances and just over $23 \mathrm{~km} / \mathrm{h}$ at shorter distances, see Table 7.

Table 7. Vehicle speeds $(\mathrm{km} / \mathrm{h}) 12 \mathrm{~m}$ before and at the pedestrian crossing depending on short or long distance between speed cushion and pedestrian crossing. (PCR = pedestrian crossing).

\begin{tabular}{llrrrr}
\hline & & \multicolumn{3}{c}{ Shorter distance } & Longer distance \\
\hline Dragonstigen & mean & $12 \mathrm{~m}$ before PCR & On PCR & $12 \mathrm{~m}$ before PCR & On PCR \\
& std dev (mean) & 24.2 & 23.1 & 17.0 & 22.1 \\
& 1.51 & 1.21 & 0.59 & 0.51 \\
& $90 \%$ & 34 & 30 & 23 & 27 \\
& $\mathrm{n}$ & 37 & 34 & 52 & 53 \\
\hline Tessins Väg & mean & 23.6 & 23.9 & 23.8 & 22.5 \\
& std dev (mean) & 0.68 & 0.65 & 0.54 & 0.43 \\
& $90 \%$ & 29 & 30 & 30 & 28 \\
& $\mathrm{n}$ & 39 & 45 & 117 & 132 \\
\hline
\end{tabular}

The differences in both traffic directions was not significant at some sites, but a longer distance between speed cushion and pedestrian crossing nevertheless proved to have positive tendencies. Speeds $12 \mathrm{~m}$ before the speed cushion were also measured, and the results are presented in Table 7 . The speed $12 \mathrm{~m}$ before the pedestrian crossing was comparable with that on the crossing, with the exceptions of one direction at Dragonstigen where the beginning of the speed cushion is $12 \mathrm{~m}$ before the pedestrian crossing. The fact that speed $12 \mathrm{~m}$ before the crossing was the same or less than on the crossing can most 
plausible be explained by drivers starting to decelerate in order to drive over the speed cushion, and then starting to accelerate again once they had reached the crossing. Vehicle speeds are assumed to be lowest at the speed cushions.

On the comparison sites at Regementsgatan/Sergels Väg and Regementsgatan/ Skvadronsgatan, speeds $12 \mathrm{~m}$ before and at the pedestrian crossing were somewhat lower than at the sites above. On neither of the study sites was the 90th percentile higher than 30 $\mathrm{km} / \mathrm{h}$ at the crossing. Vehicle speeds $12 \mathrm{~m}$ before the crossings were comparable with the sites above.

Table 8. Vehicle speeds $(\mathrm{km} / \mathrm{h}) 12 \mathrm{~m}$ before and on the pedestrian crossing at comparison sites. (PCR = pedestrian crossing).

\begin{tabular}{llrr}
\hline & & \multicolumn{2}{c}{ 'normal distance' (i.e., 5 m) } \\
& & 12 m before PCR & On PCR \\
\hline Regementsgatan/Sergels Väg & mean & 22.4 & 19.2 \\
& std dev (mean) & 0.57 & 0.59 \\
& $90 \%$ & 28 & 24.0 \\
& $\mathrm{n}$ & 72 & 56 \\
\hline Regementsgatan/Skvadronsgat & mean & 24.3 & 21.6 \\
& std dev (mean) & 0.90 & 1.43 \\
& $90 \%$ & 32 & 28 \\
& $\mathrm{n}$ & 37 & 25 \\
\hline
\end{tabular}

Speed measurements at Hultagatan were carried out using radar and at the time of measurement did not differentiate between the two driving directions, which did not give sufficient accuracy for stating speed at the pedestrian crossing or in different directions. Average speed was $32.4 \mathrm{~km} / \mathrm{h}$, (standard deviation for the mean is $1.5 \mathrm{~km} / \mathrm{h}, \mathrm{n}=22$, 90th percentile was $40.9 \mathrm{~km} / \mathrm{h}$ ). Speeds were higher at the Hulta site because the speed cushions are lower than on the sites in Malmö.

\section{CONCLUSI ONS AND DISCUSSION}

Different distances between speed cushions and pedestrian crossings in this study did not significantly affect mobility for any group of pedestrians or cyclists. However, some significant differences were shown, indicating a longer distance between speed cushions and pedestrian crossings. All pedestrians were given way by the first car from the left significantly more often, all adult and child cyclists were more often given way by cars from the left, and all cyclists were more often given way by the first car from the left, when the distance between the speed cushion and the pedestrian or cycle crossing was greater.

Average motor vehicle speed was approximately $22-23 \mathrm{~km} / \mathrm{h}$ on the study sites in Malmö, and 90th percentiles were around $30 \mathrm{~km} / \mathrm{h}$, which means that the objective of the layouts, motor vehicle speeds below $30 \mathrm{~km} / \mathrm{h}$ was met. No significant differences were measured in the different vehicle directions. The differences in both traffic directions were not significant at some sites, but a longer distance between speed cushion and pedestrian crossing still produced lower speeds. Since the speed cushion in Borås was lower than that in Malmö, speeds were higher, with an average of over $30 \mathrm{~km} / \mathrm{h}$, but measurement accuracy was not equally good. In general, however, it is reasonable to assume that vehicle speed on a pedestrian crossing depends mainly on speed cushion height, provided the distance to the speed cushion is not too great.

The fact that cyclists are also given way on cycle crossing cannot clearly be considered as an effect of traffic safety measures when, according to traffic regulations, it is cyclists who should give way to other vehicles when they cross the road at a cycle crossing. It is also important to remember that what is a long distance to pedestrian crossing can be a short 
distance for cycle crossing when the both areas often lie parallel to one another. More data to describe cyclist safety and accessibility on cycle crossings is needed.

Since motor vehicle speeds were low on the pedestrian crossings irrespective of the distance between cushion and crossing, i.e. one or two car lengths, and that it has been shown to have certain positive effects concerning accessibility for unprotected road users when the distance was two car lengths, it is suggested that this distance should be used when installing new speed cushions in the future.

\section{Acknowledgments}

This survey was financed by Skyltfonden (the Road Administration's "Road-Sign Fund"). The work took place with the kind cooperation of Malmö Highways Department, with special thanks to Olle Thor and Karin Caesar. It is our hope that the survey will provide a useful basis for safety and accessibility for pedestrians and cyclists, especially children and the elderly.

\section{REFERENCES}

Ashton, S.J . (1982). Vehicle Design and Pedestrian Injuries. I: Pedestrian Accidents. In. Chapman, A.J ., Wade, F.M., Foot, H.C. (Eds), J ohn Wiley \& Sons Ltd.

Elvik, R., Vaa, T., (2004). The Handbook of Road Safety Measures. Elsevier Science Ltd. based on Elvik, R., Mysen, A.B., Vaa, T., 1997 Traffic Safety Handbook, Institute of Transport Economics, Oslo.

J ohansson, C., (2001). Towards a Method of Improving Road Safety for Pedestrians and Cyclists, Especially in Child Pedestrian Environments. A Case Study in Borås, Sweden. Luleå Technical University. LIC 2001:29.

Teichgräber, W. (1983) The Significance of Speed for Traffic Safety. Journal of Traffic Safety, Vol 2, II Quarter.

Waltz, F.H., Hoefliger, M., Fehlmann, W. (1983). Speed Limit Reduction from 60 to $50 \mathrm{~km} / \mathrm{h}$ and Pedestrian Injuries. In: Twenty-Seventh Step Car Crash Conference Proceedings. International Research Council on Biokinetics of Impacts (IRCOBI). Society of Automotive Engineers, Warrendale, PA, pp. 311-318. 